\title{
Effect of zinc concentration in Mueller-Hinton agar on susceptibility of Pseudomonas aeruginosa to
} meropenem

Meropenem, like imipenem, is a highly potent carbapenem antibiotic active against a broad range of bacteria including Pseudomonas aeruginosa [1]. A study by Cooper and colleagues reported that zinc concentration in commercial media influenced the susceptibility of $P$. aeruginos $a$ and other gram-negative bacilli to imipenem [2]. Similar results were reported by Daly et al. in 1997 [3]. We attempted to determine whether higher zinc concentrations in Mueller-Hinton agar (MHA) influence the zone diameters of meropenem and imipenem in the disk diffusion method.

The zinc concentration in the Oxoid MHA (CM337B) used throughout the study was $0.45 \mathrm{mg} / \mathrm{L}$ having been quantified by Unipath (Basingstoke, Hants) by atomic absorption spectroscopy. The zinc ion concentration of the medium was increased to $3 \mathrm{mg} / \mathrm{L}$ by the addition of zinc acetate which gave a similar concentration to the $2.6 \mathrm{mg} / \mathrm{L}$ which was determined for BBL MHA (H2DWFX) [2].

Sixteen $P$. aeruginosa isolates were studied; the control strain used throughout the study was $P$. aeruginosa ATCC27853. All $P$. aeruginosa isolates were adjusted to an optical density of a 9.5 McFarland standard $\left(10^{8} \mathrm{cfu} / \mathrm{ml}\right)$ with sterile saline and then further diluted to achieve a final bacterial concentration of $10^{7} \mathrm{cfu} / \mathrm{ml}$. The antibiotic susceptibilities of $P$. aeruginosa isolates to meropenem (disk content $10 \mu \mathrm{g}$ ) and imipenem (disk content $10 \mu \mathrm{g}$ ) were determined with both media. The depth of MHA was $c .4 \mathrm{~mm}$. The procedure for the disk diffusion test was that recommended by the NCCLS [4].

The results of the susceptibility testing of $16 P$. aeruginosa isolates to meropenem and imipenem on Oxoid MHA and zinc-supplemented Oxoid MHA are shown in Table 1. Student's $t$-test was used in the evaluation of the difference between the susceptibilities of clinical isolates of $P$. aeruginosa to meropenem and imipenem on both media; $\mathrm{p}<0.05$ was considered to be significant.

When zinc-supplemented MHA was used, the zone diameters of meropenem and imipenem with the $16 P$. aeruginosa isolates and the control organism decreased $(p<0.05)$. Similar experiments were carried out for ceftazidime and piperacillin. No statistically significant differences were observed for these on
Table 1. Susceptibility of $16 P$ aeruginosa isolates to meropenem, imipenem, ceftazidime and piperacillin on (media containing two different amounts of zinc)

\begin{tabular}{lcc}
\hline & \multicolumn{2}{c}{ Mean (SEM) zone diameter with } \\
\cline { 2 - 3 } Antibiotic & $\begin{array}{c}\text { MHA } \\
\text { (zinc } 0.45 \mathrm{mg} / \mathrm{L})\end{array}$ & $\begin{array}{c}\text { MHA with } \mathrm{Zn} \\
\text { supplement }(3 \mathrm{mg} / \mathrm{L})\end{array}$ \\
\hline${ }^{*}$ Meropenem & $26.5(1.8)$ & $23.4(1.0)$ \\
${ }^{\dagger}$ Imipenem & $22.0(1.6)$ & $19.4(1.8)$ \\
${ }^{\ddagger}$ Ceftazidime & $20.9(1.7)$ & $21.6(1.4)$ \\
${ }^{\$}$ Piperacillin & $21.7(1.3)$ & $22.0(1.6)$ \\
\hline
\end{tabular}

${ }^{*} \mathrm{p}<0.05 ;{ }^{\dagger} \mathrm{p}<0.05 ;{ }^{\ddagger} \mathrm{p}>0.05 ;{ }_{\mathrm{p}} \mathrm{p}>0.05$.

either medium $(p>0.05)$. This effect was confirmed as specific for carbapenems only.

These observations have been explained in a recent study by Baxter and Lambert who showed that free zinc ions in aqueous solution hydrolyse imipenem and that the rate of hydrolysis depends on time and zinc concentration [5].

It is clear that, as for imipenem, the zinc concentration in MHA affects the activity of meropenem against $P$. aeruginosa. These observations may result in false reports of resistance to meropenem and imipenem and restrict the use of these antipseudomonal antibiotics in the treatment of $P$. aeruginosa infection.

S. Atmaca, Department of Microbiology, Faculty of Medicine, Dicle University, 21280 Diyarbakir, Turkey.

\section{References}

1. Sanders CC, Sanders WE, Thomson KS, Iaconis JP. Meropenem activity against resistant Gram-negative bacteria and interactions with $\beta$-lactamases. J Antimicrob Chemother 1989; 24 Suppl A: $187-196$.

2. Cooper GL, Louie A, Baltch et al. Influence of zinc on Pseudomonas aeruginosa susceptibilities to imipenem. J Clin Microbiol 1993; 31: 2366-2370.

3. Daly JS, Dodge RA, Glew RH, Soja DT, Deluca BA, Hebert S. Effect of zinc concentration in Mueller-Hinton agar on susceptibility of Pseudomonas aeruginosa to imipenem. J Clin Microbiol 1997; 35: 1027-1029.

4. National Committee for Clinical Laboratory Standards. Performance Standards for Antimicrobial Disk Susceptibility Test, 5 edn. (Approved Standard M2-A5) Villonova, PA, NCCLS. 1993.

5. Baxter IA, Lambert PA. The effect of zinc on imipenem. $J$ Antimicrob Chemother 1997; 39: 838-839. 